\title{
2 Familiarity and preference for pitch probability profiles
}

\author{
3 Anja-Xiaoxing Cui ${ }^{1} \cdot$ Meghan J. Collett $^{1} \cdot$ Niko F. Troje $^{1} \cdot$ Lola L. Cuddy $^{1}$
}

\begin{abstract}
We investigated familiarity and preference judgments of participants toward a novel musical system. We exposed participants to tone sequences generated from a novel pitch probability profile. Afterward, we either asked participants to identify more familiar or we asked participants to identify preferred tone sequences in a twoalternative forced-choice task. The task paired a tone sequence generated from the pitch probability profile they had been exposed to and a tone sequence generated from another pitch probability profile at three levels of distinctiveness. We found that participants identified tone sequences as more familiar if they were generated from the same pitch probability profile which they had been exposed to. However, participants did not prefer these tone sequences. We interpret this relationship between familiarity and preference to be consistent with an inverted U-shaped relationship between knowledge and affect. The fact that participants identified tone sequences as even more familiar if they were generated from the more distinctive (caricatured) version of the pitch probability profile which they had been exposed to suggests that the statistical learning of the pitch probability profile is involved in gaining of musical knowledge.
\end{abstract}

Keywords Music cognition - Pitch probability profile . Statistical learning $\cdot$ Preference $\cdot$ Familiarity

Anja-Xiaoxing Cui a.cui@queensu.ca

1 Department of Psychology, Queen's University, Kingston, ON, Canada

\section{Introduction}

Because affect for music is likely related to the violation and confirmation of expectancy (Meyer 1956), music psychologists have been motivated to investigate the origins of listeners' expectancies. It has been argued that the expectancies are based on the knowledge of statistical regularities in music, which we acquire unintentionally (without intention) by being exposed to the music (Huron 2006; Tillmann et al. 2000). The mere exposure effect proposed by Zajonc (1968) relates affect for music to exposure directly. In essence, it proposes that exposure alone leads to "attitude enhancement" (Zajonc 1968). This suggests that affect for music is dependent on the amount of exposure. If expectancies are based on the knowledge of music, and if we assume that exposure to music leads to knowledge about this music, then both Meyer (1956) and Zajonc (1968) link what we like to what we know. Exposure could lead to the knowledge of statistical regularities (for instance, through unintentional learning). The statistical regularities could be the basis for expectancies formed by the listener. The tension a listener feels from violation and confirmation of those expectancies leads to changes in affect. The hypothesized relationships between exposure, knowledge of statistical regularities, expectancies, and affect are visualized in Fig. 1. The dashed arrow denotes the relationship proposed by Zajonc (1968). The mere exposure effect assumes change in affect through change in exposure. Meyer (1956) and later Huron (2006) proposed that affect is related to violation and confirmation of expectancies. This is denoted by the dotted arrow. The first question we were interested in exploring is whether participants are able to gain knowledge of statistical regularities through exposure.

Some have argued that the mere exposure effect is a form of implicit memory, i.e., that what we like is basically

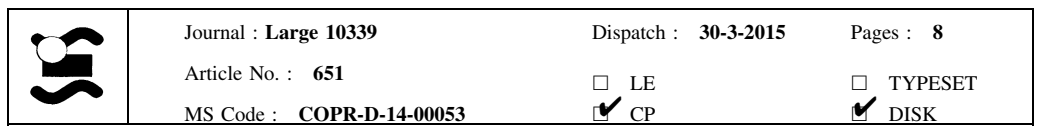


Fig. 1 Hypothesized relationship between exposure, knowledge of statistical regularities, expectancies, and affect

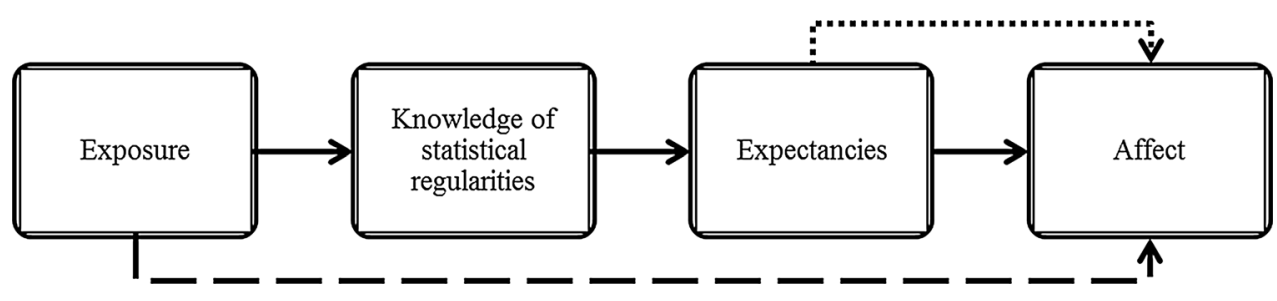

what we know (Seamon et al. 1995; Peretz et al. 1998). This suggests that asking listeners about their affect for music assesses their memory implicitly, while asking listeners about their knowledge of music would do so explicitly. This is the second question we wanted to explore in our study. However, in a recent study by Loui et al. (2010), dissociation between knowledge and affect was reported. In this study, participants "recognized" previously unheard music fashioned after the same statistical rules that were also found in the music that they had heard, but did not display a mere exposure effect. In a study by Kuhn and Dienes (2005), the opposite pattern was observed. After exposure to a tone sequence set generated using specific rules, participants liked tone sequences generated using the same rules more than tone sequences that violated those rules. However, when asked to differentiate between tone sequences that followed the rules and tone sequences that violated the rules, participants were unable to do so. Both these studies provide arguments against the proposition that the mere exposure effect is a form of implicit memory as familiarity, and liking ratings were different. On the other hand, in a study by Schellenberg et al. (2008), it was found that liking as well as familiarity ratings increase linearly as a function of exposure if participants listen to music unintentionally. Simultaneously, recognition ratings for the music increased with exposure, therefore suggesting a link between mere exposure effect and memory. But liking of music followed an inverted U-shaped function of exposure if listeners focused on the music. An inverted U-shaped relationship between stimulus complexity and liking has been proposed before by Berlyne (1974). It could be argued that stimulus complexity declines with exposure, as participants become increasingly familiar with stimuli after exposure, such that overexposure leads to boredom (Cantor 1968). Huron (2006) proposes that predictability in music, which would require familiarity with the music obtained through exposure, leads to positive responses. However, high predictability might also lead to negative appraisal, if the listener feels as though he or she is being "treated as musical children" (Huron 2006, p. 141).

It should be noted that the form of the relationship between variables like stimulus complexity or novelty and preference varies between different stimulus domains
(Martindale et al. 1990). While the study by Schellenberg et al. (2008) mentioned above found an inverted U-shaped function of exposure to musical pieces and preference, one can find positive and negative linear functions of acoustic qualities such as loudness or pitch (Martindale et al. 1990), and other factors, such as meaningfulness of the stimuli, rather than stimuli complexity, may contribute to preference ratings to a greater degree (Martindale and Moore 1989).

To address the questions of (a) whether participants are able to acquire knowledge about statistical regularities of a musical system after exposure, and (b) whether results differ if we assess participants memory implicitly or explicitly, we exposed participants to music from a pre-defined musical system. To circumvent influence by what participants already know, we assessed participants on their knowledge of and affect for novel musical systems. The statistical regularities of the systems that we used were described by Huron and Veltman (2006). Unlike the statistical regularities underlying the musical system used by Loui et al. (2010), the statistical regularities of the new musical system used here were only represented in terms of their first-order statistics, that is, as the probability distribution of pitch occurrences, without accounting for the sequence in which they occur. First-order probability profiles have been used in algorithms used to determine the musical system of a piece (Krumhansl 1990; Huron and Veltman 2006). First-order probability profiles based on real music also seem to correspond to the mental hierarchy of pitch classes (Krumhansl 1985).

Another variable we sought to modify is the listener's expectancies. By altering the first-order probability profile such that it varies in distinctiveness, we hoped to manipulate the amount of violation and confirmation of potentially formed expectancies. We created three levels of distinctiveness. The standard level of distinctiveness corresponded to the probability profile described by Huron and Veltman (2006). By sharpening the probability profile, we created a profile with a high level of distinctiveness. By flattening the probability profile, we created a profile with a low level of distinctiveness. Note that these manipulations kept the rank order of pitch occurrence intact. We exposed participants to tone sequences at the standard level of distinctiveness. Afterward, we tested either the

$\begin{array}{lll}\text { Journal : Large 10339 } & \text { Dispatch : 30-3-2015 } & \text { Pages : } 8 \\ \text { Article No. : } \mathbf{6 5 1} & \square \text { LE } & \square \text { TYPESET } \\ \text { MS Code : COPR-D-14-00053 } & \boldsymbol{\sim}_{\mathrm{CP}} & \checkmark \text { DISK }\end{array}$


participants' knowledge of or their affect for tone sequences at all levels of distinctiveness in a two-alternative forced-choice task. We did this by either asking participants to indicate which melody they found more familiar (knowledge) or which melody they preferred (affect). Expectancies formed during exposure (i.e., based on music at the standard level of distinctiveness) are more likely to be confirmed if the participant is tested with tone sequences at the high level of distinctiveness: Pitch classes occurring often at the standard level, which are the pitch classes that the participant would expect, occur even more often at the high level of distinctiveness. These expectancies are more likely to be violated if the participant is tested with tone sequences at the low level of distinctiveness: Pitch classes occurring often at the standard level, which are the pitch classes that the participant would expect, occur less at the low level of distinctiveness. The question we addressed with this manipulation is whether distinctiveness influences participants' responses.

By creating two experimental groups that differed in the instructions they received, we aimed to test whether any knowledge that might be acquired during exposure can be acquired unintentionally, i.e., without the intention to learn. Participants were focused on the musical stimuli in both conditions. However, while participants in the group with more instructions were told that they would be asked questions about the music they were about to hear, i.e., about the two-alternative forced-choice task, participants in the group with less instructions were not told about that phase in the experiment. Participants in the group with less instructions thus had no reason to actively try to learn about the music during the exposure phase.

While the mere exposure effect offers no direct prediction, one could argue that participants have not been exposed to tone sequences at levels of distinctiveness other than the standard level prior to the testing of knowledge or affect. This would mean lower preference for tone sequences at levels of distinctiveness other than the standard level. However, if participants acquire knowledge of statistical regularities and generalize the hierarchy of pitch classes, tone sequences at high levels of distinctiveness could be regarded as more familiar. Researchers who have proposed that the mere exposure effect is a form of implicit memory would then assume that those tone sequences lead to higher preference. A different pattern is expected if there is indeed dissociation between knowledge and affect.

\section{Methods}

\section{Stimuli}

Tone sequences were generated from a version of Temperley's (2007) pitch model using pitch profiles of two novel musical systems. The two novel musical systems used in this study were two Gregorian chant modes: Hypophrygian and Lydian. The pitch profile of these modes has been described by Huron and Veltman (2006). Huron and Veltman (2006) tallied the pitch classes occurring in a sample of the Liber Usualis, a book containing over 2000 Gregorian chants. Three versions of pitch profiles were constructed by raising the profile described by Huron and Veltman (2006) to different exponents using an algorithm by Smith and Schmuckler (2004). An exponent of 1 created the standard level of distinctiveness. An exponent of 2 created the high level of distinctiveness. An exponent of .5 created the low level of distinctiveness. Figure 2 visualizes the pitch profile for both modes at each level of distinctiveness. A profile at the high level of distinctiveness is characterized by higher "peaks" and lower "dips." A profile at the low level of distinctiveness is "flatter" than profiles at higher levels of distinctiveness. Each tone sequence was $15 \mathrm{~s}$ long and consisted of 50 tones of equal duration. Tones were of a flute-like timbre. Tone sequences were generated by independently drawing from the probability profiles. The range of pitches used for the tone
Fig. 2 Pitch probability profiles for a Hypophrygian and for b Lydian tone sequences. The low level of distinctiveness is indicated with dotted lines. The standard level of distinctiveness is indicated with dashed lines. The high level of distinctiveness is indicated with solid lines
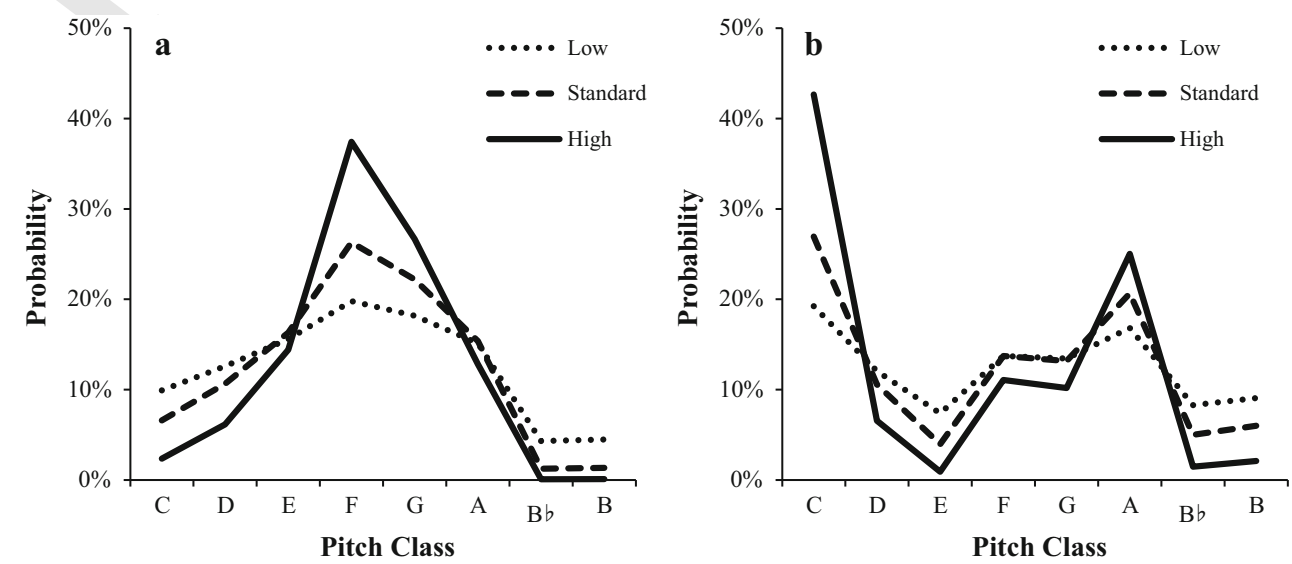

\begin{tabular}{|l|lll|}
\hline Journal : Large 10339 & Dispatch : 30-3-2015 & Pages : $\mathbf{8}$ \\
Article No. : $\mathbf{6 5 1}$ & $\square$ LE & $\square$ TYPESET \\
MS Code : COPR-D-14-00053 & $\sim_{\text {CP }}$ & $\checkmark$ DISK \\
\hline
\end{tabular}


Fig. 3 Excerpts from sample tone sequences

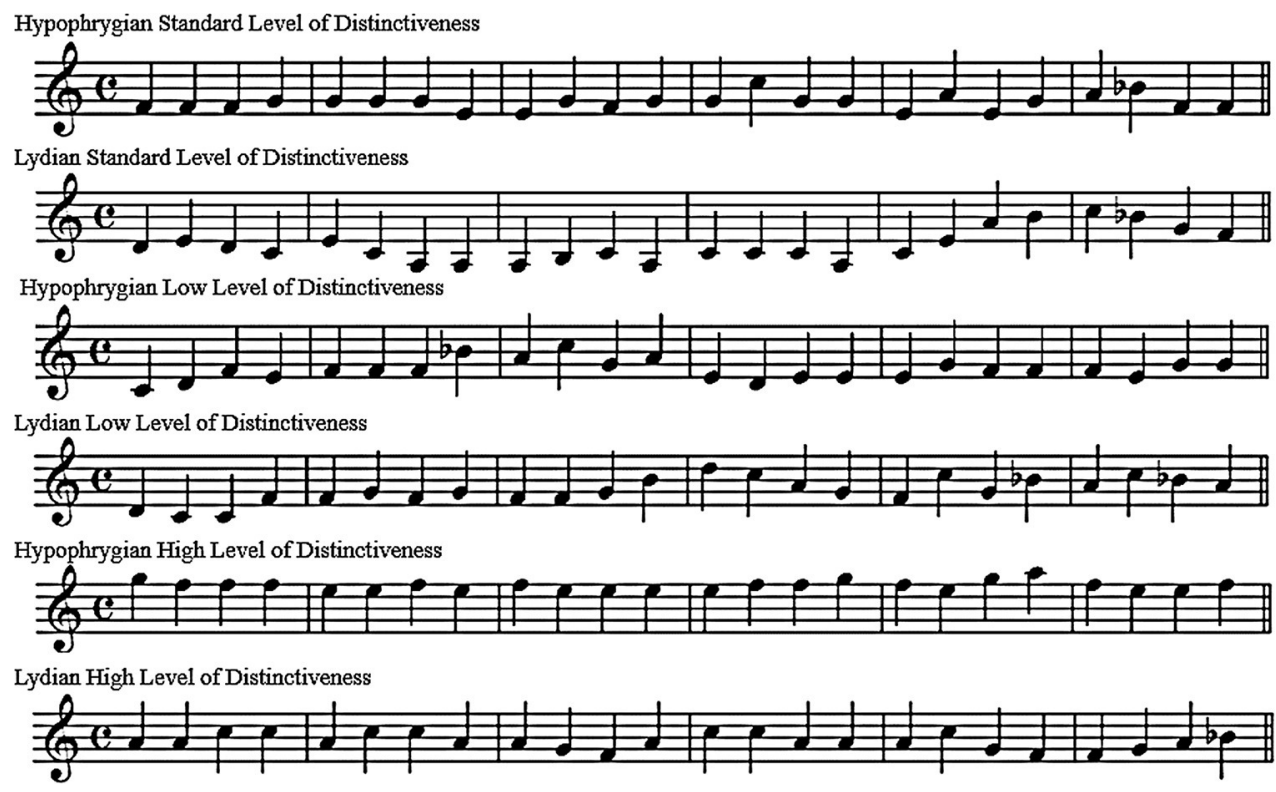

sequences was determined by a normal probability density function with a $\mu_{1}$, which was drawn from a normal distribution with $\mu_{2}=68$ and $\sigma_{2}=3.63$, and $\sigma_{1}=5.39$, with 68 corresponding to a central pitch of $\mathrm{G} \#^{4}$ and the interval of $\mu_{2} \pm \sigma_{1}$ spanning the range from $\mathrm{D} \sharp^{4}$ to $C \sharp^{5}$. An excerpt from example tone sequences for each of the six probability profiles (Hypophrygian standard, high, and low level of distinctiveness, Lydian standard, high, and low level of distinctiveness) can be found in Fig. 3 .

\section{Participants}

Eighty-two participants with less than five years of formal music training were recruited from Queen's University and compensated monetarily for their time. Twenty-eight of those participants were male, 54 female.

\section{Procedure and Design}

Testing took place in a sound-attenuated chamber. Participants were instructed to adjust the volume to their preferred level. Testing was divided into two phases. In the first phase (the exposure phase), participants were exposed to tone sequences generated from the pitch profile of one mode (exposed mode) at the standard level of distinctiveness. The mode (either Hypophrygian or Lydian) was chosen randomly. The exposure phase consisted of 100 individual tone sequences. The exposure phase lasted $25 \mathrm{~min}$. The ensuing second phase (the test phase) comprised 30 trials of a two-alternative forced-choice task. Tone sequences presented during the test phase were new, i.e., participants had not heard them prior. We paired a tone sequence from the exposed mode and a tone sequence generated from the non-exposed mode. There were 10 such pairings (trials) at each of the three levels of distinctiveness; thus, there were a total of 30 trials. The order in which the 30 trials were presented was randomized. The level of distinctiveness was our within-subject factor.

There were two between-subject factors yielding four experimental groups. The first factor was instruction: participants were either informed at the beginning of the experiment, that the test phase would take place (with instruction), or they were told to follow instructions "as they come along" (no instruction). The latter group was thus unaware of the test phase until it started. The second factor divided participants into a familiarity and a preference group. In the test phase, the familiarity group was asked to indicate which tone sequence they found more familiar. The preference group was asked to indicate which tone sequence they preferred. Nineteen participants were in the familiarity-no instruction group, 23 participants were in the familiarity-with instruction group, 20 participants were in the preference-no instruction group, and 20 participants were in the preference-with instruction group.

\section{Results}

The dependent variables were calculated by dividing the number of times the tone sequence of the exposed mode was chosen in the test phase by the number of trials at each level of distinctiveness. The choice of the exposed mode was classified as "correct." The numbers are expressed as a percentage of correct responses in Fig. 3. Thus, a percent "correct" of $50 \%$ would indicate chance performance. There was no effect of "instruction," $F(1,78)=1.10$,

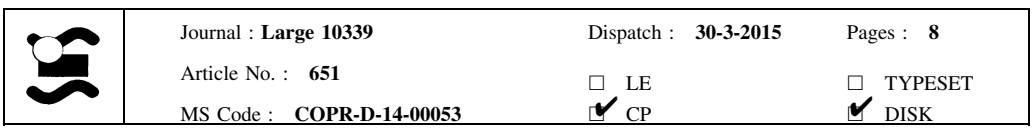




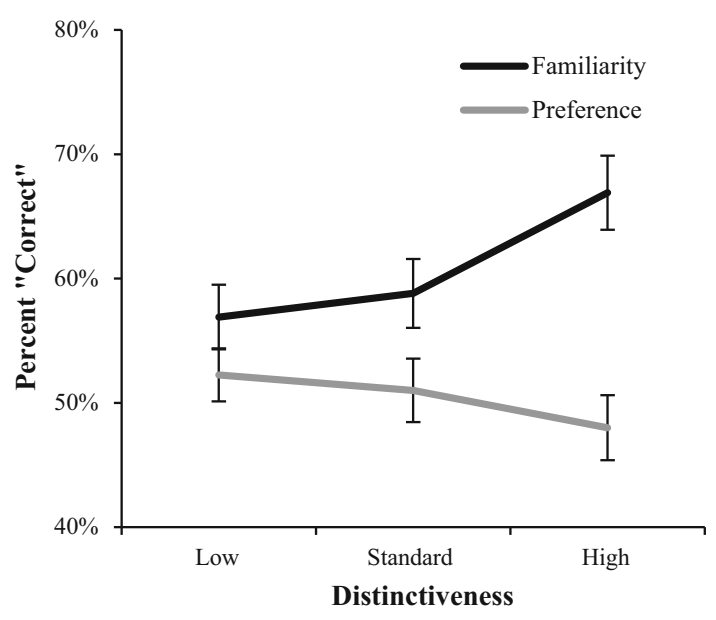

Fig. 4 Percent "correct" for familiarity and preference group at each level of distinctiveness. The choice of the exposed mode was classified as "correct." Error bars depict the standard error of mean

285

286

287

288

289

290

291

292

293

294

295

296

297

298

299

300

301

302

303 $p=.297$, and no interaction effects involving "instruction," $p>.05$, so data were pooled across this factor for further analysis. There was a main effect of familiarity/ preference, $F(1,80)=19.24, p<.001$, such the percent "correct" scores of participants in the familiarity group were higher than the percent "correct" scores of participants in the preference group. Participants in the familiarity group performed higher than chance (one-sample $t$-tests, low distinctiveness $t(41)=2.65, p=.011$, standard distinctiveness $t(41)=3.18, p=.003$, high distinctiveness $t(41)=5.67, \quad p<.001)$, but those in the preference group did not $(p s>.05)$. Moreover, there was a significant interaction between familiarity/preference and distinctiveness, $F(2,160)=4.57, p=.012$, and no significant main effect of distinctiveness, $F(2,160)=0.81$, $p=.447$. The linear contrast for distinctiveness in the familiarity group was significant $(F(1,41)=6.11, p=.018)$, but not in the preference group $(F(1,39)=1.70, p=.200)$. The data are depicted in Fig. 4.

\section{Discussion}

The participants in the familiarity group indicated that tone sequences generated from the pitch probability profile of the mode that they had previously been exposed to were more familiar. Thus, it can be argued that they generalized the pitch probability profile they were exposed to: participants indicated familiarity for tone sequences generated from the same pitch probability profile as the sequences heard during exposure. The only similarity between the tone sequences participants had heard and the tone sequences in the test phase is that they were generated from the same pitch probability profile. As there was no effect of "instruction," this demonstrates successful unintentional learning of a first-order probability profile, i.e., learning that took place without the intention to learn. Although the participants in the "with instruction" group were not told that the tone sequences in the test phase would be similar to the tone sequences in the exposure phase, it can be argued that participants may have suspected this. Participants could then have tried to actively "learn" the pitch probability profile. The participants in the "no instruction" group were told to follow instructions "as they come along." As they were not told about the test phase, we assume that any learning that they exhibited took place unintentionally. Moreover, participants in the familiarity group chose tone sequences from the pitch probability profile of the exposed mode at the high level of distinctiveness more often than chance. These tone sequences followed the statistical rules set forth during exposure more distinctly. Participants in the familiarity group also chose tone sequences from the pitch probability profile of the exposed mode at the low level of distinctiveness. However, this effect was less pronounced than when tone sequences were generated at the standard or high level of distinctiveness. The significant linear contrast such that the effect increased with levels of distinctiveness can be described as a "caricature effect": the caricatured version of the probability profile (the probability profile at the high level of distinctiveness) appears to be more familiar. This indicates that participants generalized the probability profile and supported the theory that salient pitches are important for acquisition of pitch profiles and the tonal hierarchy (Krumhansl and Cuddy 2010). In future studies, more levels of distinctiveness could be tested to validate this idea of a "caricature effect." It should be noted that based on our data, the representation of the distribution is not absolute, so judgments of familiarity are facilitated by the caricature of the distribution. It is not impossible that participants abstracted an interval distribution rather than a pitch distribution. We believe that our argument of distinctiveness would hold nonetheless, as the distribution of intervals would become more distinct at the high level of distinctiveness of the pitch distribution and less distinct at the low level of distinctiveness of the pitch distribution.

The absence of a significant linear contrast of distinctiveness in the preference group, the fact that their behavior in the test phase was not different from chance, and particularly the strong interaction between distinctiveness and familiarity/preference do not suggest a positive linear relationship between knowledge and affect. This goes against the prediction made by mere exposure effect theories: in our experiment, mere exposure did not lead to "attitude enhancement" (Zajonc 1968). This also does not evidently support the idea that affect assessment assesses implicit memory (Seamon et al. 1995; Peretz et al. 1998). However, 
the findings in our study could be interpreted as assessing implicit memory if the relationship between knowledge and affect follows an inverted U-shaped curve.

Szpunar et al. (2004) for instance have suggested that liking follows an inverted U-shaped function of exposure. Participants in our study might have been overexposed to the pitch probability profile, thereby increasing familiarity, but decreasing preference. This decrease was especially visible for tone sequences at the high level of distinctiveness. If exposure is positively correlated with familiarity, then the data could be interpreted as lying on the right part of an inverted U-shaped relationship. Figure 5 graphs the data shown in Fig. 4 in a different fashion: in this figure, the mean percentages in the familiarity group are plotted against the mean percentages in the preference group. As can be seen, the interpretation of the data as lying on the right shoulder of an inverted U-shaped function of exposure could be considered: the more familiar the stimuli were, the less they were preferred. If stimulus complexity declines with familiarity, the interpretation of the data as lying on the left shoulder of an inverted $U$ shape of stimulus complexity could be considered (Berlyne 1974). This would also be consistent with the boredom effect described by Cantor (1968).

Another perspective from which to view our results is in reference to schema and prototype theory. Smith and Melara (1990) propose that listeners may understand a musical phrase by treating it as a categorization problem. In our experiment, then the standard probability profiles are the prototypes to which new tone sequences are assimilated. Tone sequences generated from the low level of

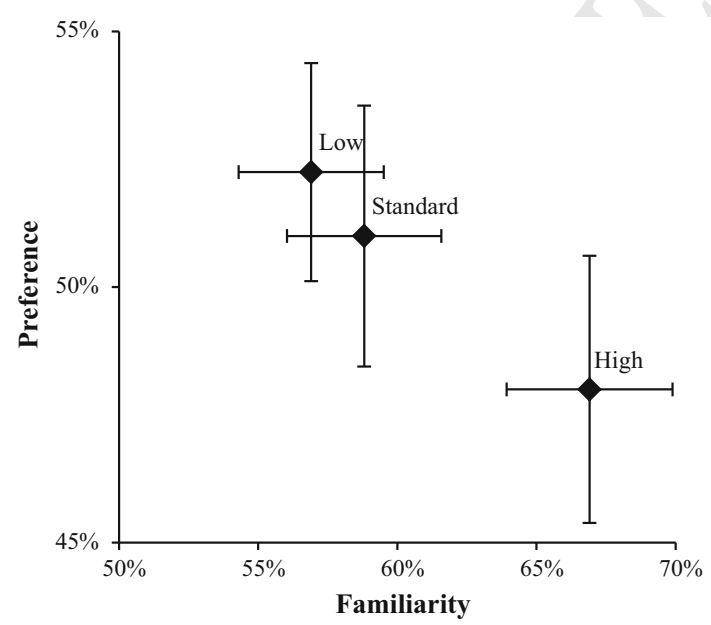

Fig. 5 Percent "correct" at each level of distinctiveness. The percent "correct" of the familiarity group is plotted against the percent "correct" of the preference group for each level of distinctiveness. The horizontal and vertical error bars depict standard error of the mean for the familiarity group and for the preference group, respectively distinctiveness profile require greater effort to be assimilated and thus appear less familiar. In an experiment by Smith and Melara (1990), participants rated musical phrases with varying degrees of prototypicality of Western music. Musical phrases with a greater degree of prototypicality were rated as less unusual by musically untrained participants. For the same participants, ratings of pleasantness were negatively related to unusualness and positively related to prototypicality. Assuming preference and pleasantness are positively related, and familiarity and unusualness are negatively related, our results stand in contrast to the pattern for these participants reported. The same experiment, however, was run using highly trained participants also. In contrast to the musically untrained participants, and in accordance with our data, these participants rated tone sequences as more pleasant when they had a lower degree of prototypicality and were rated as more unusual. It could be argued then that participants in our experiment gained proficiency during exposure with the novel musical system such that their pattern was similar to the pattern found using Western music and highly trained students.

Schellenberg et al. (2008) found that the liking of music follows an inverted U-shaped function of exposure if listeners focus on the music, whereas liking increases linearly as a function of exposure if participants listen to the music incidentally. At first glance, this result might not be compatible with our proposition. However, the incidental condition in the study by Schellenberg et al. (2008) exposed participants to the music, while they had to press keys and count the number of occurrences of words in a story that they were listening to simultaneously to the music. This led to lower recognition ratings in the incidental group. Thus, participants in the incidental group might not have been overexposed to the music yet, leading to the observed linear relationship between exposure and liking. The data from the study by Schellenberg et al. (2008) could be lying on the left part of an inverted $\mathrm{U}$-shaped function of exposure.

It should be noted that the stimuli used in the studies described above (Loui et al. 2010; Schellenberg et al. 2008; Szpunar et al. 2004) were different from the stimuli used in our experiment. In a series of three experiments, Szpunar et al. (2004) used short tone sequences of 5-9 piano tones and excerpts of "classical" music pieces. The number of occurrences of those stimuli was varied to manipulate exposure. Thus, stimuli were repeated. Schellenberg et al. (2008) used excerpts of "classical" music pieces. Again, the number of occurrences of those excerpts was changed to manipulate exposure. These two studies differ from ours and the studies by Kuhn and Dienes (2005) and Loui et al. (2010) in that they manipulated exposure by repeating stimuli. Furthermore, Schellenberg et al. (2008) and

$\begin{array}{lll}\text { Journal : Large 10339 } & \text { Dispatch : 30-3-2015 } & \text { Pages : } 8 \\ \text { Article No. : } \mathbf{6 5 1} & \square \text { LE } & \square \text { TYPESET } \\ \text { MS Code : COPR-D-14-00053 } & \boldsymbol{\sim}_{\text {CP }} & \boldsymbol{L I S K}_{\text {DISK }}\end{array}$


Szpunar et al. (2004) were not interested in the ability of participants to passively learn a new musical system.

Loui et al. (2010) generated tone sequences from a novel musical system as we did. However, they used the BohlenPierce scale. The statistical regularities in their system were based on transitional probabilities. The stimuli in the experiments reported by Kuhn and Dienes (2005) used non-local dependencies. However, our study used Gregorian chant modes defined by first-order probabilities. Both Kuhn and Dienes (2005) and Loui et al. (2010) claim that participants in their studies were able to generalize the rules on which the music was based. When explicitly asked to do so, participants in the study by Kuhn and Dienes (2005) were unable to differentiate between tone sequences that were similar to the tone sequence set to which they had been exposed and tone sequences that violated the rules on which the other tone sequences had been based. However, they differentiated between those two types of tone sequences when asked how much they liked them. On the other hand, in the experiments by Loui et al. (2010), and similarly in our study, participants with little or no music training (less than five years) were able to generalize the statistical rules when asked to recognize tone sequences or when asked about their familiarity with the tone sequences. But while recognition or familiarity ratings indicate that participants passively learned about a new musical system, Loui et al. (2010) found no preference for previously heard tone sequences or tone sequences composed in the exposed style over tone sequences following another set of transitional probabilities. Similarly, we found no preference for tone sequences composed in the exposed mode over tone sequences of a different mode. Furthermore, there was no significant linear contrast of distinctiveness in the preference group. So, even though the tone sequences were more or less violating potentially formed expectancies, there was no difference in affect. These differences in the observed pattern between the different studies may be explained when considering the time for which participants were exposed to the tone sequences that were supposed to set forth the rules. While our participants and the participants in the study by Loui et al. (2010) were exposed to the novel music system for about $25 \mathrm{~min}$, participants in the study by Kuhn and Dienes (2005) were "trained" on the rules for six min. The shorter exposure could mean that the boredom effect (Cantor 1968) has not set in yet.

In conclusion, it can be said that our results support the idea that passive statistical learning of probability profiles could be involved in gaining musical knowledge, as participants indicated that tone sequences generated from a probability profile at the high level of distinctiveness, i.e. a "caricatured" version of the probability profile to which they were exposed, were most familiar. Participants in our study did not prefer tone sequences that other participants found familiar after the same amount of exposure. Future explorations might manipulate the length of exposure to determine whether participants did not prefer the tone sequences because they have been overexposed.

Acknowledgments This study was supported by a Discovery Grant from the Natural Sciences and Engineering Council of Canada to LLC. We would like to thank four anonymous reviewers for their helpful comments.

\section{References}

Berlyne DE (1974) The new experimental aesthetics. In: Berlyne DE (ed) Studies in the new experimental aesthetics: steps towards an objective psychology of aesthetic appreciation. Hemisphere Publishing Co., Washington, pp 1-25

Cantor GN (1968) Children's "like-dislike" ratings of familiarized and unfamiliarized visual stimuli. J Exp Child Psychol 6:651-657

Huron (2006) Sweet anticipation. MIT Press, Cambridge

Huron D, Veltman J (2006) A cognitive approach to medieval mode: evidence for an historical antecedent to the major/minor system. Empir Musicol Rev 1:33-55

Krumhansl CL (1985) Perceiving tonal structure in music. Am Sci 73:371-378

Krumhansl CL (1990) Cognitive foundations of musical pitch. Oxford University Press, New York

Krumhansl CL, Cuddy LL (2010) A theory of tonal hierarchies in music. In: Jones MR, Fay RR, Popper AN (eds) Music perception. Springer, New York, pp 51-87

Kuhn G, Dienes Z (2005) Implicit learning of nonlocal musical rules: implicitly learning more than chunks. J Exp Psychol Learn Mem Cogn 31:1417-1432

Loui P, Wessel DL, Hudson Kam CL (2010) Humans rapidly learn A01 536 grammatical structure in a new musical scale. Music Percept 327-377

Martindale C, Moore K (1989) Relationship of musical preference to collative, ecological, and psychophysical variables. Music Percept 431-445

Martindale C, Moore K, Borkum J (1990) Aesthetic preference: Anomalous findings for Berlyne's psychobiological theory. Am J Psychol 53-80

Meyer LB (1956) Emotion and meaning in music. University of Chicago Press, Chicago

Peretz I, Gaudreau D, Bonnel AM (1998) Exposure effects on music preference and recognition. Mem Cognit 26:884-902

Schellenberg EG, Peretz I, Vieillard S (2008) Liking for happy-and sad-sounding music: effects of exposure. Cogn Emot 22:218-237

Seamon JG, Williams PC, Crowley MJ, Kim IJ, Langer SA, Orne PJ, Wishengrad DL (1995) The mere exposure effect is based on implicit memory: effects of stimulus type, encoding conditions, and number of exposures on recognition and affect judgments. J Exp Psychol Learn Mem Cogn 21:711-721

Smith JD, Melara RJ (1990) Aesthetic preference and syntactic prototypicality in music: $T$ is the gift to be simple. Cognition 34:279-298

Smith NA, Schmuckler MA (2004) The perception of tonal structure through the differentiation and organization of pitches. J Exp Psychol Hum Percept Perform 30:268-286 
Szpunar KK, Schellenberg EG, Pliner P (2004) Liking and memory for musical stimuli as a function of exposure. J Exp Psychol Learn Mem Cogn 30:370-381

Temperley D (2007) Music and probability. MIT Press, Cambridge Tillmann B, Bharucha JJ, Bigand E (2000) Implicit learning of tonality: a self-organizing approach. Psychol Rev 107:885-913
Zajonc RB (1968) Attitudinal effects of mere exposure. J Pers Soc Psychol 9:1-27

\begin{tabular}{|l|lll|}
\hline Journal : Large 10339 & Dispatch : 30-3-2015 & Pages : $\mathbf{8}$ \\
Article No. : $\mathbf{6 5 1}$ & $\square$ LE & $\square$ TYPESET \\
MS Code : COPR-D-14-00053 & $\sim_{\mathrm{CP}}$ & $\boldsymbol{\sim}$ DISK \\
\hline
\end{tabular}

